\title{
High-Resolution Spectroscopy of the Transiting Planet Host Star TrES-1
}

\section{Citation}

Sozzetti, Alessandro, David Yong, Guillermo Torres, David Charbonneau, David W. Latham, Carlos Allende Prieto, Timothy M. Brown, Bruce W. Carney, and John B. Laird. 2004. "HighResolution Spectroscopy of the Transiting Planet Host Star TrES-1." The Astrophysical Journal 616 (2): L167-70. https://doi.org/10.1086/426864.

\section{Permanent link}

http://nrs.harvard.edu/urn-3:HUL.InstRepos:41397383

\section{Terms of Use}

This article was downloaded from Harvard University's DASH repository, and is made available under the terms and conditions applicable to Other Posted Material, as set forth at http:// nrs.harvard.edu/urn-3:HUL.InstRepos:dash.current.terms-of-use\#LAA

\section{Share Your Story}

The Harvard community has made this article openly available.

Please share how this access benefits you. Submit a story.

Accessibility 


\title{
HIGH-RESOLUTION SPECTROSCOPY OF THE TRANSITING PLANET HOST STAR TrES-1
}

\author{
Alessandro Sozzetti, ${ }^{1,2}$ David Yong,${ }^{3}$ Guillermo Torres, ${ }^{2}$ David Charbonneau, ${ }^{2}$ David W. Latham, ${ }^{2}$ \\ Carlos Allende Prieto, ${ }^{4}$ Timothy M. Brown, ${ }^{5}$ Bruce W. Carney, ${ }^{3}$ and John B. Laird ${ }^{6}$ \\ Received 2004 September 25; accepted 2004 October 19; published 2004 October 28
}

\begin{abstract}
We report on a spectroscopic determination of the stellar parameters and chemical abundances for the parent star of the transiting planet TrES-1. Based on a detailed analysis of iron lines in our Keck and Hobby-Eberly Telescope spectra, we derive $T_{\text {eff }}=5250 \pm 75 \mathrm{~K}, \log g=4.6 \pm 0.2$, and $[\mathrm{Fe} / \mathrm{H}]=0.00 \pm 0.09$. By measuring the Ca II activity indicator and by putting useful upper limits on the $\mathrm{Li}$ abundance, we constrain the age of TrES-1 to be $2.5 \pm 1.5$ Gyr. By comparing theoretical stellar evolution models with the observational parameters, we obtain $M_{\star}=0.89 \pm 0.05 M_{\odot}$ and $R_{\star}=0.83 \pm 0.05 R_{\odot}$. Our improved estimates of the stellar parameters are utilized in a new analysis of the transit photometry of TrES-1 to derive a mass $M_{p}=(0.76 \pm 0.05) M_{\mathrm{J}}$, a radius $R_{p}=$ $1.04_{-0.05}^{+0.08} R_{\mathrm{J}}$, and an inclination $i=89.5_{-1.3}^{+0.5} \mathrm{deg}$. The improved planetary mass and radius estimates provide the grounds for new crucial tests of theoretical models of evolution and evaporation of irradiated extrasolar giant planets.
\end{abstract}

Subject headings: planetary systems: formation — stars: abundances — stars: individual (TrES-1)

Online material: color figure

\section{INTRODUCTION}

The discovery of an extrasolar Jupiter-sized planet transiting the disk of the K0 V star TrES-1 (Alonso et al. 2004) has marked the first success of ground-based photometric surveys targeting large areas of the sky with small-size telescopes searching for low-amplitude periodic variations in the light curves of bright stars $(V \leq 13)$. In this Letter we report on a detailed spectroscopic determination of the stellar parameters and chemical abundances of iron and lithium of the transiting planet host star.

TrES-1 is only the second transiting planet orbiting a star bright enough to allow for a variety of follow-up analyses similar to those conducted for HD 209458b (see, e.g., Charbonneau 2003 and references therein). In particular, high-resolution, high signalto-noise ratio spectroscopic observations of TrES-1 can be readily conducted, allowing for improved values of the effective temperature, surface gravity, and metallicity of the star. These parameters, when compared with stellar evolution models, provide the means to derive good estimates of its mass and radius. Finally, by combining spectroscopic observations with transit photometry, it is possible to determine refined estimates of the mass and radius of the planet. A better knowledge of these parameters offers us the tantalizing possibility for making crucial tests of giant planet formation, migration, and evolution. In addition, the hypothesis of self-enrichment due to the recent ingestion of planetary material can be tested using detailed abundances of elements such as lithium.

\footnotetext{
${ }^{1}$ Department of Physics and Astronomy, University of Pittsburgh, 100 Allen Hall, 3941 O'Hara Street, Pittsburgh, PA 15260.

${ }^{2}$ Harvard-Smithsonian Center for Astrophysics, 60 Garden Street, Cambridge, MA 02138; asozzett@cfa.harvard.edu, gtorres@cfa.harvard.edu, dcharbon@cfa.harvard.edu,dlatham@cfa.harvard.edu.

${ }^{3}$ Department of Physics and Astronomy, University of North Carolina, CB 3255 Phillips Hall, Chapel Hill, NC 27599-3255; yong@physics.unc.edu, bruce@physics.unc.edu.

${ }^{4}$ McDonald Observatory and Department of Astronomy, University of Texas, Austin, TX 78712; callende@ hebe.as.utexas.edu.

${ }^{5}$ High Altitude Observatory/National Center for Atmospheric Research, 3450 Mitchell Lane, Boulder, CO 80307; timbrown@hao.ucar.edu.

${ }^{6}$ Department of Physics and Astronomy, Bowling Green State University, 104 Overman Hall, Bowling Green, OH 43403; laird@tycho.bgsu.edu.
}

\section{DATA REDUCTION AND ABUNDANCE ANALYSIS}

The spectroscopic observations that led to the radial velocity confirmation of the planetary nature of the transiting object detected by the TrES (Trans-atlantic Exoplanet Survey) consortium (Alonso et al. 2004) were performed with the High Resolution Echelle Spectrometer (HIRES) and its $\mathrm{I}_{2}$ absorption cell on the Keck I telescope (Vogt et al. 1994). Eight star+iodine spectra and one template spectrum were collected over 18 days in 2004 July. The template spectrum used here has a resolution $R \simeq$ 65,000 and a signal-to-noise ratio $\mathrm{S} / \mathrm{N} \simeq 80$ pixel $^{-1}$. Three $R \simeq 60,000$ spectra were taken with the High Resolution Spectrograph (HRS; Tull 1998) on the Hobby-Eberly Telescope (HET) during consecutive nights in 2004 August, with spectral coverage in the range 5879-7838 $\AA$. The averaged spectrum has $\mathrm{S} / \mathrm{N} \simeq 120 \mathrm{pixel}^{-1}$. For all spectra, thorium-argon lamp exposures provided the wavelength calibration.

Our abundance analysis of the Keck/HIRES and HET/HRS spectra of TrES-1 was carried out under the assumption of standard local thermodynamic equilibrium (LTE) using a modified version of the spectral synthesis code MOOG (Sneden 1973) and a grid of Kurucz (1993) stellar atmospheres. We selected a set of $30 \mathrm{Fe}$ I and four Fe II lines (with lower excitation potentials $0.86 \leq \chi_{l} \leq 5.03 \mathrm{eV}$ and $2.58 \leq \chi_{l} \leq$ $3.90 \mathrm{eV}$, respectively) from the HIRES spectrum and used standard packages in IRAF to derive equivalent widths (EWs) for all of them. Our selection of lines and transition probabilities followed that of Lee \& Carney (2002).

\section{STELLAR PARAMETERS}

Alonso et al. (2004) report on the analysis of seven spectra of TrES-1 taken with the CfA Digital Speedometers (Latham 1992). By comparing them with a library of synthetic spectra, they derive estimates of the effective temperature, surface gravity, and metallicity as follows: $T_{\text {eff }}=5250 \pm 200 \mathrm{~K}, \log g=$ $4.5 \pm 0.5$, and $[\mathrm{Fe} / \mathrm{H}] \sim 0.0$. As the authors later point out in their discovery paper, these relatively large uncertainties have a significant impact on the final estimates of the mass and radius of the transiting planet, and ultimately on the possibility to confront theory with observation. We describe below the fourfold strategy we have devised to better constrain the stellar and con- 


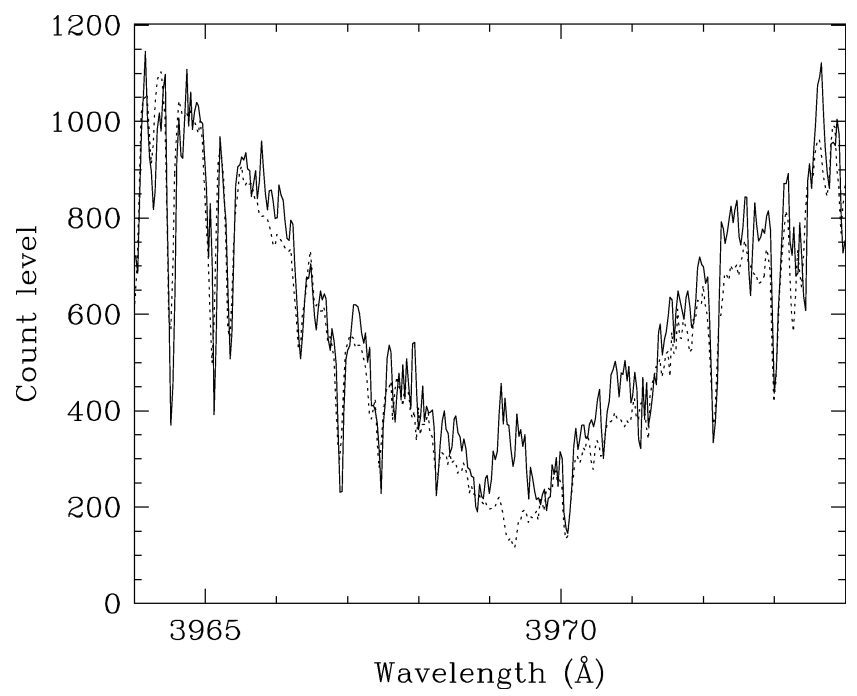

FIG. 1.-Comparison between the $\mathrm{Ca}$ II $\mathrm{H}$ line for TrES-1 (solid line) and an inactive star of the same temperature (dotted line).

sequently planetary parameters, based on a detailed analysis of our Keck and HET spectra.

\subsection{Atmospheric Parameters from Spectroscopy}

The stellar atmospheric parameters for TrES-1 were obtained using a standard technique of $\mathrm{Fe}$ ionization balance (see, e.g., Santos et al. 2004 and references therein). The best-fit configuration of the parameters is obtained by means of an iterative procedure that encompasses the following three steps: (1) the effective temperature is obtained by imposing abundances $\log \epsilon(\mathrm{Fe} \mathrm{I})$ obtained from the $\mathrm{Fe}$ I lines independent of the lower excitation potential $\chi_{l}$ (i.e., zero correlation coefficient); (2) the microturbulent velocity $\xi_{t}$ is determined so that $\log \epsilon(\mathrm{Fe} \mathrm{I})$ is independent of the reduced equivalent widths $\mathrm{EW}_{\lambda} / \lambda$; and (3) the surface gravity is determined by forcing exact agreement between abundances derived from the $\mathrm{Fe}$ II and those obtained from the Fe I lines. Uncertainties in the parameters were estimated following the prescriptions of Neuforge \& Magain (1997) and Gonzalez \& Vanture (1998) and rounded to $25 \mathrm{~K}$ in $T_{\text {eff }}, 0.1 \mathrm{dex}$ in $\log g$, and $0.05 \mathrm{~km} \mathrm{~s}^{-1}$ in $\xi_{t}$.

The final atmospheric parameters, along with the best-fit Fe I abundances, are $T_{\text {eff }}=5250 \pm 75 \mathrm{~K}, \log g=4.6 \pm$ $0.2, \xi_{t}=0.95 \pm 0.1 \mathrm{~km} \mathrm{~s}^{-1}$, and $[\mathrm{Fe} / \mathrm{H}]=0.00 \pm 0.09$. Including in the analysis EWs of $11 \mathrm{Fe}$ I and four Fe II lines measured in the region of the HRS-averaged spectrum in common with the Keck template spectrum gave identical results. The good agreement between the HIRES-only and HIRES + HRS analysis suggests that significant sources of systematic errors due to different spectrograph/configurations are absent. Our spectroscopic estimates of $T_{\text {eff }}, \log g$, and $[\mathrm{Fe} / \mathrm{H}]$ also agree remarkably well with the numbers reported by Alonso et al. (2004).

Finally, a possible matter of concern are systematic uncertainties inherent in the one-dimensional, LTE model atmospheres used in the derivation of effective temperature and metallicity for a star as cool as TrES-1. However, recent studies (Yong et al. 2004) argue that non-LTE effects arise primarily in stars significantly cooler than TrES-1 and with metallicities significantly departing from solar (e.g., Asplund 2003 and references therein).

\section{2. $T_{\text {eff }}$ Estimate from Photometry}

There is no direct distance estimate for TrES-1, and this prevents the determination of an absolute luminosity. In addition, as the star does not show signs of evolution off the main sequence, the surface gravity constitutes a poor constraint for the models. The effective temperature and metallicity, together with an age estimate as we discuss below, are thus the only reliable metrics that can be used in the comparison with theoretical isochrones. It is then crucial to provide an independent estimate of $T_{\text {eff }}$ for an assessment of the validity of its spectroscopic value and uncertainty.

To this end, we utilized the $B V J H K$ apparent magnitudes to derive several $T_{\text {eff }}$ estimates based on a number of empirical color-temperature calibrations from the literature. The Johnson $B$ and $V$ magnitudes for TrES- 1 were obtained by means of differential photometry (including differential extinction) relative to a number of nearby stars (G. Mandushev 2004, private communication). For the apparent luminosity in the infrared filters, we relied on the 2MASS catalog. From these values, and their relative uncertainties, we derived empirical $T_{\text {eff }}$ estimates based on the color-temperature calibrations of MartínezRoger et al. (1992), Alonso et al. (1996), and Ramírez \& Meléndez (2004). We obtained an average value for the effective temperature of $T_{\text {eff }}=5206 \pm 92 \mathrm{~K}$. This result is in good agreement with our own spectroscopic $T_{\text {eff }}$ determinations reported in the previous section, giving us confidence in the spectroscopically determined $T_{\text {eff }}$ from Keck and HET.

\subsection{Stellar Activity and Age}

Inspection of the $\mathrm{Ca}$ II $\mathrm{H}$ and $\mathrm{K}$ lines in the HIRES spectra revealed a slight core reversal, indicating that TrES-1 is an active star. In Figure 1 we show a region of the HIRES template spectrum centered on the $\mathrm{Ca}$ II $\mathrm{H}$ line. For comparison, we have overplotted the spectrum of an old, inactive star (HIP 86830) with the same temperature from the Sozzetti et al. (2004) sample of metal-poor stars, which has a metallicity $[\mathrm{Fe} / \mathrm{H}]=-0.68$. The emission feature is clearly evident. A possible explanation for the activity level is that the star is not very old. To provide useful constraints on its age, we have collected three different pieces of external evidence.

First, we have measured the chromospheric activity index $S$ from the $\mathrm{Ca} \mathrm{H}$ and $\mathrm{K}$ lines, based on the prescriptions by Duncan et al. (1991), for TrES-1 and for two Hyades stars (HD 28462 and HD 32347) of the same spectral type observed in the context of the G-dwarf Planet Search program (Latham 2000). We transformed our $S$-values to the standard Mount Wilson $S$ index, applying the relation derived by Paulson et al. (2002). We then applied relations from Noyes et al. (1984 and references therein) to convert to the chromospheric emission ratio $R_{\mathrm{HK}}^{\prime}$ and to derive estimates of the age $t$ for the three stars. We obtained $\log R_{\mathrm{HK}}^{\prime}=-4.77$ for TrES-1. The activity levels for the two Hyades stars are in excellent agreement with the values reported by Paulson et al. (2002), giving us confidence that our $R_{\mathrm{HK}}^{\prime}$ for TrES-1 is reliable. As its activity level is about half that of the two Hyades members, but higher than that reported for its very close analog $\alpha$ Cen B ( $\log R_{\mathrm{HK}}^{\prime}=-4.92$; Chmielewski 2000), we can argue that TrES-1 is older than Hyades stars of the same spectral type but younger than $\alpha$ Cen B ( 4.2 Gyr; Henry et al. 1996). Indeed, based on the Noyes et al. (1984) relations, we find an age value $t=2.5 \mathrm{Gyr}$. We note, however, that our measure of $R_{\mathrm{HK}}^{\prime}$ is based on a single-epoch observation; thus, additional $\mathrm{Ca}$ II measurements are clearly encouraged. 
Second, following Gonzalez (1998) and using the atmospheric parameters derived from the Fe-line analysis, we synthesized a $10 \AA$ region of the spectrum, including the $6707.8 \AA \mathrm{Li}$ line in the HRS-averaged spectrum. In Figure 2 we show the comparison of the spectrum of TrES-1 with three models, each differing only in the $\mathrm{Li}$ abundance assumed. The lithium line is not detectable by eye in the noise. We place an upper limit for the $\mathrm{Li}$ abundance of $\log \epsilon(\mathrm{Li})<0.1$, consistent with the star being older than a Hyades star of the same temperature.

Finally, due to a fast decrease in activity levels for ages greater than $\sim 1$ Gyr (Pace \& Pasquini 2004), at 2 Gyr the emission in $\mathrm{Ca}$ II is down by a factor of a few with respect to the Hyades, and the value of $R_{\mathrm{HK}}^{\prime}$ for TrES-1 is consistent with this interpretation. Our preliminary age estimate for TrES-1 is then $2.5 \pm 1.5 \mathrm{Gyr}$.

\subsection{Stellar Mass and Radius}

Using the constraints described above on $T_{\text {eff }},[\mathrm{Fe} / \mathrm{H}]$, and the age of the star, we used stellar evolution models and ran Monte Carlo simulations to infer the stellar mass and radius for TrES-1 as well as their uncertainties. The simulations were repeated using two different evolutionary models: Girardi et al. (2000) and Yi et al. (2003). We obtain very good agreement in the derived parameters in both cases, to within 0.01 solar units in both mass and radius, despite slightly different assumptions in the input physics of these two sets of models. We report here the results for TrES-1 using the Girardi et al. (2000) models: $M_{\star}=0.89 \pm 0.05 M_{\odot}, R_{\star}=0.83 \pm 0.05 R_{\odot}$. These error bars include a somewhat arbitrary contribution of 0.04 added in quadrature to the formal Monte Carlo errors of 0.03 in both mass and radius, to account for unforeseen systematics in both the stellar evolution and stellar atmosphere models, as well as other physical assumptions. The uncertainties on metallicity and effective temperature contribute about equally to the errors on both mass and radius, while the age uncertainty contributes negligibly.

Finally, based on our simulations, we estimate an absolute magnitude $M_{v}=5.85 \pm 0.15$ and thus place TrES- 1 at a nominal distance $d \simeq 150 \mathrm{pc}$. Its resulting Galactic velocity vector is $[U, V, W]=[-15,-33,16] \mathrm{km} \mathrm{s}^{-1}$.

\section{PLANETARY PARAMETERS}

Following Alonso et al. (2004), we utilized the revised values of $M_{\star}$ and $R_{\star}$, and their uncertainties, in a new $\chi^{2}$ minimization procedure of the photometric points for the model light curve as a function of $R_{p}$ and $i$. We find $R_{p}=1.04_{-0.05}^{+0.08} R_{\mathrm{J}}$ and $i=$ $89.5_{-1.3}^{+0.5} \mathrm{deg}$. The values and uncertainties for the primary mass, inclination, and the radial velocity semiamplitude are then combined to give a value of the planetary mass $M_{p}=(0.76 \pm$ 0.05) $M_{\mathrm{J}}$. With the improved value of the stellar mass, now the dominant contribution to the planet mass uncertainty comes from the spectroscopic orbit, while the contribution due to the error in the inclination angle is negligible.

While the revised value for $M_{p}$ is essentially identical to the one reported by Alonso et al. (2004), the planetary radius found here is slightly smaller because of the smaller stellar radius estimate obtained in our analysis.

\section{SUMMARY AND DISCUSSION}

We have derived new values of the stellar atmospheric parameters of the transiting planet host-star TrES-1 from high-
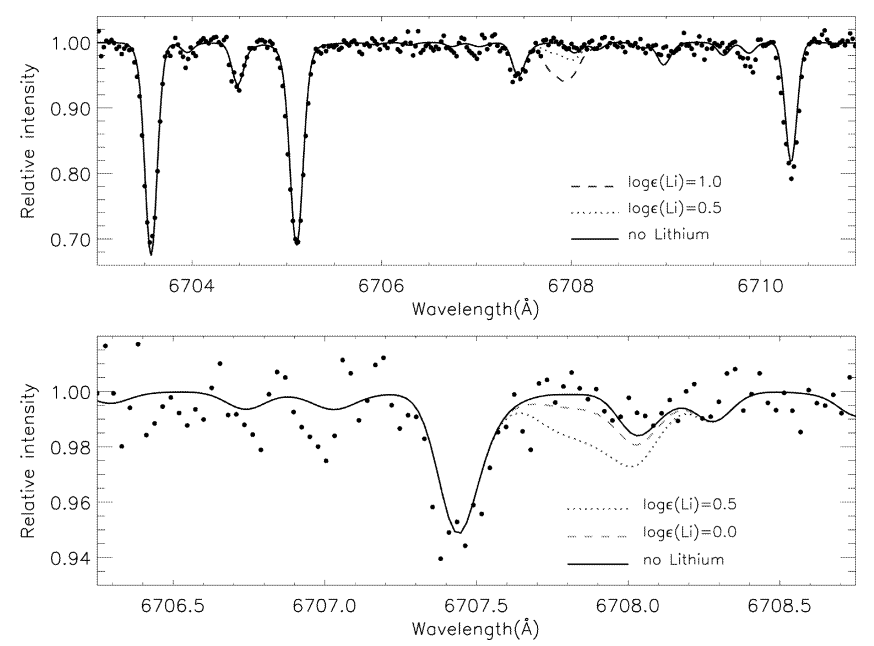

FIG. 2.-Portion (top panel) of the HET-averaged spectrum of TrES-1 containing the $\mathrm{Li}$ I line at $6707.8 \AA$ (filled circles), compared to three syntheses (lines of various colors and styles), each differing only for the lithium abundance assumed. The bottom panel shows the data on an enlarged scale, so the model spectra for low $\mathrm{Li}$ abundance can be distinguished. [See the electronic edition of the Journal for a color version of this figure.]

resolution, high $\mathrm{S} / \mathrm{N}$ spectra. Our spectroscopic $T_{\text {eff }}$-values are in very good agreement with empirical color-temperature calibrations and with the estimates reported by Alonso et al. (2004). The star is a main-sequence object with a metallicity consistent with solar. The lack of detectable lithium argues both against youth and recent pollution events due to ingestion of planetary material. Its measured Ca II activity levels are further suggestive of an object with an age of a few gigayears, an intermediate value between the Hyades and $\alpha$ Cen B.

Based on the new constraints on atmospheric parameters and age, we have significantly improved on the determination of the stellar and planetary mass and radius. Our updated values for $M_{\star}$ and $R_{\star}$ are consistent with those reported by Alonso et al. (2004), although we find a slightly smaller radius and a slightly larger mass. The most significant improvement comes at the level of the determination of $R_{p}$ and $M_{p}$ with our new analysis of the transit photometry. In Figure 3 we show masses and radii for all transiting planets known to date, including TrES-1. Thanks to the more accurate values of the stellar parameters, the radius and mass of the planet have reduced uncertainties with respect to those reported by Alonso et al. (2004). In particular, for very similar values of $M_{p}$, the difference between the values of $R_{p}$ for HD 209458 and TrES-1, about $25 \%$, is now significant at the $3 \sigma$ level.

The well-determined radius of TrES-1 opens the door to new important tests of theoretical evolutionary models of irradiated extrasolar giant planets (e.g., Bodenheimer et al. 2003; Burrows et al. 2004; Chabrier et al. 2004), as its parent star is about $1000 \mathrm{~K}$ cooler than HD 209458 and OGLE-TR-56, the other two objects that have recently been targets of extensive investigations. Its radius seems to agree better with the predictions from models that do not invoke additional heat/power sources in the core (Showman \& Guillot 2002; Baraffe et al. 2003) or tidal heating effects due to the gravitational perturbation of an undetected long-period companion (Bodenheimer et al. 2003). Given its orbital radius and the characteristics of the host star, its mass-loss rate (Baraffe et al. 2004) is also likely to be significantly reduced with respect to those, for example, expected for OGLE-TR-56b and OGLE-TR-132b. 


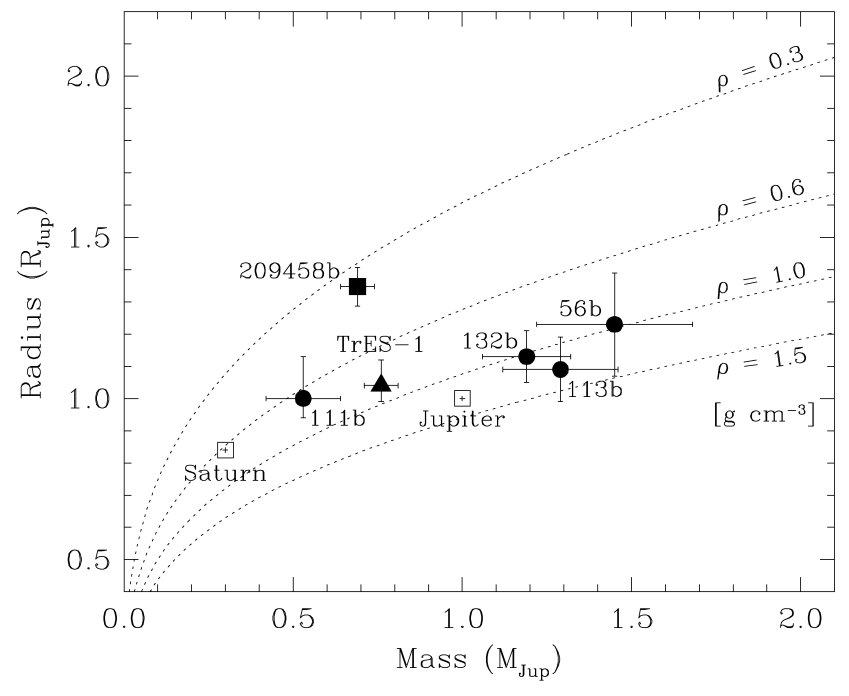

FIg. 3.-Radius vs. mass for the sample of transiting extrasolar planets discovered to date, including TrES-1 (this work). The values and uncertainties are taken from Brown et al. (2001) for HD 209458b, Torres et al. (2004) for OGLE-TR-56b, Bouchy et al. (2004) for OGLE-TR-113b, Moutou et al. (2004) for OGLE-TR-132b, and Pont et al. (2004) for OGLE-TR-111b.
Additional detections of giant planets transiting relatively bright stars covering a range of spectral types are clearly necessary for a continued improvement of our understanding of the structure and evolutionary properties of this peculiar class of objects.

Over the course of this investigation, we have benefited from stimulating discussions with various colleagues, in particular, G. Mandushev, R. Alonso, H. Deeg, J. Belmonte, E. Dunham, and F. O'Donovan. We are thankful to the Director of the McDonald Observatory, D. Lambert, for providing Director's discretionary time and to the HET staff, in particular S. Odewahn, V. Riley, and M. Villareal, for their assistance. The Hobby-Eberly Telescope is operated by the McDonald Observatory on behalf of the University of Texas at Austin, Pennsylvania State University, Stanford University, Ludwig Maximillians Universität München, and Georg August Universität Göttingen. Some of the data presented herein were obtained at the W. M. Keck Observatory, which is operated as a scientific partnership among the California Institute of Technology, the University of California, and the National Aeronautics and Space Administration.

\section{REFERENCES}

Alonso, A., Arribas, S., \& Martinez-Roger, C. 1996, A\&A, 313, 873

Alonso, R., et al. 2004, ApJ, 613, L153

Asplund, M. 2003, IAU Highlights of Astronomy, Vol. 13, ed. E. Engvold, in press

Baraffe, I., Chabrier, G., Barman, T., Allard, F., \& Hauschildt, P. H. 2003, A\&A, 402, 701

Baraffe, I., Selsis, F., Chabrier, G., Barman, T. S., Allard, F., Hauschildt, P. H., \& Lammer, H. 2004, A\&A, 419, L13

Bodenheimer, P., Laughlin, G., \& Lin, D. N. C. 2003, ApJ, 592, 555

Bouchy, F., Pont, F., Santos, N. C., Melo, C., Mayor, M., Queloz, D., \& Udry, S. 2004, A\&A, 421, L13

Brown, T. M., Charbonneau, D., Gilliland, R. L., Noyes, R. W., \& Burrows, A. 2001, ApJ, 552, 699

Burrows, A., Hubeny, I., Hubbard, W. B., Sudarsky, D., \& Fortney, J. J. 2004, ApJ, 610, L53

Chabrier, G., Barman, T., Baraffe, I., Allard, F., \& Hauschildt, P. H. 2004, ApJ, 603, L53

Charbonneau, D. 2003, in ASP Conf. Ser. 294, Scientific Frontiers in Research on Extrasolar Planets, ed. D. Deming \& S. Seager (San Francisco: ASP), 449

Chmielewski, Y. 2000, A\&A, 353, 666

Duncan, D. K., et al. 1991, ApJS, 76, 383

Girardi, L., Bressan, A., Bertelli, G., \& Chiosi, C. 2000, A\&AS, 141, 371

Gonzalez, G. 1998, A\&A, 334, 221

Gonzalez, G., \& Vanture, A. D. 1998, A\&A, 339, L29

Henry, T. J., Soderblom, D. R., Donahue, R. A., \& Baliunas, S. L. 1996, AJ, 111,439

Kurucz, R. 1993, CDROM 13, ATLAS9 Stellar Atmosphere Programs and $2 \mathrm{~km} / \mathrm{s}$ Grid (Cambridge: SAO)
Latham, D. W. 1992, in IAU Colloq. 135, Complementary Approaches to Double and Multiple Star Research, ed. H. A. McAlister \& W. I. Hartkopf (ASP Conf. Ser. 32; San Francisco: ASP), 110

Latham, D. W. 2000, in ASP Conf. Ser. 219, Disks, Planetesimals, and Planets, ed. F. Garzón, C. Eiroa, D. de Winter, \& T. J. Mahoney (San Francisco: ASP), 596

Lee J.-W., \& Carney, B. W. 2002, AJ, 124, 1511

Martínez-Roger, C., Arribas, S., \& Alonso, A. 1992, Mem. Soc. Astron. Italiana, 63, 263

Moutou, C., Pont, F., Bouchy, F., \& Mayor, M. 2004, A\&A, 424, L31

Neuforge-Verheecke, C., \& Magain, P. 1997, A\&A, 328, 261

Noyes, R. W., Hartmann, L. W., Baliunas, S. L., Duncan, D. K., \& Vaughan, A. H. 1984, ApJ, 279, 763

Pace, G., \& Pasquini, L. 2004, A\&A, 426, 1021

Paulson, D. B., Saar, S. H. Cochran, W. D., \& Hatzes, A. P. 2002, AJ, 124, 572

Pont, F., Bouchy, F., Queloz, D., Santos, N. C, Melo, C., Mayor, M., \& Udry, S. 2004, A\&A, 426, L15

Ramírez, I., \& Meléndez, J. 2004, ApJ, 609, 417

Santos, N. C., Israelian, G., \& Mayor, M. 2004, A\&A, 415, 1153

Showman, A. P., \& Guillot, T. 2002, A\&A, 385, 166

Sneden, C. A. 1973, Ph.D. thesis, Univ. Texas at Austin

Sozzetti, A., Latham, D. W., Torres, G., Stefanik, R. P., Boss, A. P., Carney, B. W., \& Laird, J. B. 2004, BAAS, 36, 62.01

Torres, G., Konacki, M., Sasselov, D. D., \& Jha, S. 2004, ApJ, 609, 1071

Tull, R. G. 1998, Proc. SPIE, 3355, 387

Vogt, S. S., et al. 1994, Proc. SPIE, 2198, 362

Yi, S., Kim, Y.-C., \& Demarque, P. 2003, ApJS, 144, 259

Yong, D., Lambert, D. L., Allende Prieto, C., \& Paulson, D. B. 2004, ApJ, 603, 697 